\title{
ON SUSPENDING HOMOTOPY SPHERES
}

\author{
PETER W. HARLEY
}

A homotopy $n$-sphere is a compact topological $n$-manifold having the homotopy type of $S^{n}$ and a fake $n$-cell a compact, contractible, topological $n$-manifold whose boundary is homeomorphic to $S^{n-1}$. The object of this paper is to establish the following two propositions as regards such classes of manifolds.

THEOREM 1. The suspension of a homotopy 4-sphere is homeomorphic to $S^{5}$.

TheORem 2. The suspension of a fake 4-cell is homeomorphic to $I^{5}$.

It should be noted that M. Hirsch [4] has proved both theorems for the case in which the manifolds are smooth by the methods of differential topology. The case for piecewise linear (combinatorial) manifolds follows immediately as a result of their admitting compatible smooth structures.

We prove Theorem 2 first as follows: Let $F^{4}$ be a fake 4-cell and $h: \mathrm{Bd} F^{4} \times[0,1) \rightarrow F^{4}$ a collar for $\mathrm{Bd} F^{4}$ in $F^{4}$. Put $X=\mathrm{Cl}\left(F^{4}-h[0,1 / 2]\right)$. Note that $X$ is homeomorphic to $F^{4}$. Let $N^{5}=F^{4} \times[-2,2]$. We claim that Int $N^{5}$ is a contractible open 5 -manifold which is 1 -connected at infinity, admits a piecewise linear triangulation and thus by Stallings [6] is homeomorphic to $E^{5}$. Obviously the interior of $N^{5}$ is contractible and since by Van Kampen's theorem Bd $N^{5}$ is simply connected (it is in fact a homotopy 4 -sphere) Int $N^{5}$ is 1 -connected at infinity. It is easily seen that the double $2 N^{5}$ of $N^{5}$ is a homotopy 5 -sphere and thus by Poincaré's conjecture for topological $n$-manifolds $(n \geqq 5)$, which was proved by E. H. Connell and can be found in M. H. A. Newman [5], a 5-sphere. Hence Int $N^{5}$ is homeomorphic to an open subset of $S^{5}$. Consequently, it admits a piecewise-linear triangulation and is homeomorphic to $E^{5}$. Clearly $X \times(-1)$ and $X \times 1$ are cellular in Int $N^{5}$, for they have arbitrarily small neighborhoods homeomorphic to Int $N^{5}$. It follows (Theorem 1 of [1]) that there is a mapping $F: N^{5} \rightarrow N^{5}$ such that $F \mid \mathrm{Bd} N^{5}=$ id and the only nondegenerate inverse sets of $F$ are $X \times(-1)$ and $X \times 1$. Let $g: \operatorname{Bd} X \times[-1,1]$ $\rightarrow S(\mathrm{Bd} X)$ be the natural mapping. $F g^{-1}$ is a homeomorphism of $S(\mathrm{Bd} X)$ onto $F(\mathrm{Bd} X \times[-1,1])$. Hence $F(\mathrm{Bd} X \times[-1,1])$ is a 4-sphere. Clearly $F(\mathrm{Bd} X \times[-1,1])$ is locally flat in Int $N^{5}$ except

Presented to the Society, January 24, 1968; received by the editors June 5, 1967. 
possibly at $F(\mathrm{Bd} X \times-1)$ and $F(\mathrm{Bd} X \times 1)$. Hence by Chernavskii [3] (or by results submitted to the Annals of Mathematics by R. C. Kirby) locally flat. By the Generalized Schoenflies Theorem $F(X \times[-1,1])$ is a cell. If $G: X \times[-1,1] \rightarrow S(X)$ is the natural map (which extends $g$ ), then $F G^{-1}$ is a homeomorphism of $S(X)$ onto the 5-cell $F(X \times[-1,1])$. Since $X$ is homeomorphic to $F^{4}, S\left(F^{4}\right)$ is a 5 -cell.

Theorem 1 now follows by removing from a homotopy 4 -sphere $M^{4}$ the interior of a 4-simplex (4-cell with locally flat boundary) $\sigma^{4}$ to obtain a fake 4-cell $F^{4}$. By Theorem $1, S\left(F^{4}\right)$ is a cell. Hence $S\left(M^{4}\right)$ $=S\left(F^{4}\right) \cup S\left(\sigma^{4}\right)$ where $S\left(F^{4}\right) \cap S\left(\sigma^{4}\right)=S\left(\mathrm{Bd} \sigma^{4}\right)$ and is thus a 5 -sphere.

Theorem 2 also follows from Theorem 1 by reversing the argument above and using the following theorem of J. C. Cantrell [2].

THEOREM 3. If the $(n-1)$-sphere $(n>3) S$ in $S^{n}$ is locally collared at a point $p$ in one side and locally collared in a deleted neighborhood of $p$ on the other side, then $S$ is locally flat at $p$.

The author would like to acknowledge several helpful suggestions made by the referee.

\section{BIBLIOGRAPHY}

1. M. Brown, A proof of the generalized Schoenfies theorem, Bull. Amer. Math. Soc. 66 (1960), 74-76.

2. J. C. Cantrell, Almost locally flat embeddings of $S^{n-1}$ in $S^{n}$, Bull. Amer. Math. Soc. 69 (1963), 716-718.

3. A. J. Chernavskii, Isotopies in Euclidean spaces, Russian Math. Surveys. 19 (1964), 63-65.

4. M. W. Hirsch, "On homotopy spheres of low dimension" in Differential and combinatorial topology, Princeton Univ. Press, Princeton, N. J., 1965.

5. M. H. A. Newman, The engulfing theorem for topological manifolds, Ann. of Math. (2) 84 (1966), 555-571.

6. J. Stallings, The piecewise linear structure of euclidean spaces, Proc. Cambridge Philos. Soc. 58 (1962), 481-488.

The University of Georgia 